\title{
Sensitivity analysis for generalized quasi-variational relation problems in locally G-convex spaces
}

Nguyen Van Hung*

\section{"Correspondence:}

ngvhungdhdt@yahoo.com Department of Mathematics, Dong Thap University, 783 Pham Huu Lau Street, Ward 6, Cao Lanh City, Vietnam

\begin{abstract}
In this paper, we study generalized quasi-variational relation problems in locally G-convex spaces. Using the Kakutani-Fan-Glicksberg fixed-point theorem for upper semicontinuous set-valued mapping with nonempty closed acyclic values, we establish an existence theorem of a solution set for these problems. Moreover, the stability and closedness of the solution set for these problems are also obtained. The results presented in the paper improve and extend the main results in the literature. MSC: 47J20; 49J40

Keywords: generalized quasi-variational relation problems; G-convex spaces; Kakutani-Fan-Glicksberg fixed-point theorem; quasiconvexity; existence; closedness; upper semicontinuity; compactness
\end{abstract}

\section{Introduction and preliminaries}

The generalized quasi-variational relation problems include, as special cases, the generalized variational inclusion problems, the generalized vector equilibrium problems, the generalized vector variational inequality problems etc. In recent years, a lot of results for the existence and stability of solutions for variational relation problems, vector equilibrium problems and vector variational inequality problems have been established by many authors in different ways. For example, variational relation problems [1-4], vector equilibrium problems [5-18], vector variational inequality problems $[19,20]$ and the references therein.

For a set $X$, we shall denote by $2^{X}$ and $\langle X\rangle$ the families of all subsets of $X$ and the family of all nonempty finite subsets of $X$, respectively. For each $A \in\langle X\rangle,|A|$ denotes the cardinality of $A$. Let $\Delta_{n}$ denote the standard $n$-dimensional simplex in $R^{n+1}$ with vertices $\left\{e_{1}, e_{2}, \ldots, e_{n+1}\right\}$, that is,

$$
\Delta_{n}=\left\{u \in \mathbb{R}^{n+1}: u=\sum_{i=1}^{n+1} \lambda(u) e_{i}, \lambda(u) \geq 0, \sum_{i=1}^{n+1} \lambda(u)=1\right\},
$$

where $e_{i}$ is the $i$ th unit vector in $\mathbb{R}^{n+1}$.

For any nonempty subset $J$ of $\{0,1,2, \ldots, n\}$, we denote $\Delta_{J}$ by the convex hull of the vertices $\left\{e_{j}: j \in J\right\}$.

(c) 2012 Hung; licensee Springer. This is an Open Access article distributed under the terms of the Creative Commons Attribution License (http://creativecommons.org/licenses/by/2.0), which permits unrestricted use, distribution, and reproduction in any medium, provided the original work is properly cited. 
A convex set $A$ in a vector space is called a convex space if it is equipped with a topology which includes the Euclidean topology on convex hulls of any nonempty finite subsets of $A$.

The notion of a $G$-convex space was introduced by Park and Kim in [21]. Let $X$ be a topological space, $A \subseteq X$ be a nonempty subset and a function $\Gamma:\langle A\rangle \rightarrow 2^{X} \backslash\{\emptyset\}$ be such that the following conditions hold:

(a) for each $M, N \in\langle A\rangle, \Gamma(M) \subset \Gamma(N)$ if $M \subset N$,

(b) for each $M \in\langle A\rangle$ with $|M|=n+1$, there exists a continuous mapping $\phi_{M}: \Delta_{n} \rightarrow \Gamma(M)$ such that, for each $J \in\langle M\rangle, \phi_{M}\left(\Delta_{J}\right) \subset \Gamma(J)$, where $\Delta_{J}$ denotes the face of $\Delta_{n}$ corresponding to $J \in\langle M\rangle$.

Then $(X, A, \Gamma)$ is called a generalized convex space (or a $G$-convex space). If $A=X$, we omit $A$ simply write $(X, \Gamma)$.

For a $G$-convex space $(X, A, \Gamma)$, a subset $B$ of $X$ is said to be $G$-convex if, for each $M \in\langle A\rangle$, $M \subseteq B$ implies $\Gamma(M) \subseteq B$. A space $X$ is said to have a $G$-convex structure if and only if $X$ is a $G$-convex space. A $G$-convex $X$ is said to be a locally $G$-convex space if $X$ is a uniform topological space with uniformity $U$, which has an open base $B=\left\{V_{i}: i \in I\right\}$ of symmetric entourages such that for each $v \in B$, the set $V(x):=\{y \in X:(y, x) \in V\}$ is a G-convex set for each $x \in X$.

Now, we pass to our problem setting. Let $X, Y, Z$ be real locally $G$-convex Hausdorff topological vector spaces, $A \subseteq X, B \subseteq Y$ and $D \subseteq Z$ be nonempty compact convex subsets. Let $K_{1}: A \rightarrow 2^{A}, K_{2}: A \rightarrow 2^{A}, T: A \rightarrow 2^{B}$ be multifunctions and $R(x, z, y)$ be a relation linking $x \in A, z \in D$ and $y \in B$. We adopt the following notations (see [2]). Letters $\mathrm{w}, \mathrm{m}$ and $\mathrm{s}$ are used for weak, middle and strong kinds of considered problems respectively. For subsets $U$ and $V$ under consideration, we adopt the following notations:

$$
\begin{aligned}
& (u, v) \mathrm{w} U \times V \text { means } \forall u \in U, \exists v \in V, \\
& (u, v) \mathrm{m} U \times V \text { means } \exists v \in V, \forall u \in U, \\
& (u, v) \mathrm{s} U \times V \text { means } \forall u \in U, \forall v \in V, \\
& \rho_{1}(U, V) \text { means } U \subset V, \\
& \rho_{2}(U, V) \text { means } U \cap V \neq \emptyset, \\
& \rho_{3}(U, V) \text { means } U \not \subset V, \\
& \rho_{4}(U, V) \text { means } U \cap V=\emptyset .
\end{aligned}
$$

Let $\alpha \in\{\mathrm{w}, \mathrm{m}, \mathrm{s}\}$ and $\rho \in\left\{\rho_{1}, \rho_{2}, \rho_{3}, \rho_{4}\right\}$. We consider the following for a generalized quasivariational relation problem (in short, $\left(\mathrm{QVR}_{\alpha}\right)$ ):

$\left(\mathrm{QVR}_{\alpha}\right)$ : Find $\bar{x} \in A$ such that $\bar{x} \in K_{1}(\bar{x})$ and $(y, z) \alpha K_{2}(\bar{x}) \times T(\bar{x})$ satisfying

$$
R(\bar{x}, z, y) \text { holds. }
$$

Let $\Sigma_{\alpha}(R)$ be the solution set of $\left(\mathrm{QVR}_{\alpha}\right)$.

Special cases of the problem $\left(\mathrm{QVR}_{\alpha}\right)$ are as follows:

(I) If we let $A, D, B, X, Y, Z, K_{1}, K_{2}, T$ be as in $\left(\mathrm{QVR}_{\alpha}\right)$ and $F: A \times D \times B \rightarrow 2^{Z}$ be a multifunction, the relation $R$ is defined as follows:

$$
R(x, z, y) \text { holds iff } 0 \in F(x, z, y) .
$$


Then $\left(\mathrm{QVR}_{\alpha}\right)$ becomes the generalized quasi-variational inclusion problem:

Find $\bar{x} \in A$ such that $\bar{x} \in K_{1}(\bar{x})$ and $(y, z) \alpha K_{2}(\bar{x}) \times T(\bar{x})$ satisfying

$$
0 \in F(\bar{x}, z, y)
$$

(II) If we let $A, D, B, X, Y, Z, K_{1}, K_{2}, T, R$ be as in $\left(\mathrm{QVR}_{\alpha}\right)$ and $F: A \times D \times B \rightarrow 2^{Z}$ and $G: A \times D \rightarrow 2^{Z}$ be multifunctions, the relation $R$ is defined as follows:

$$
R(x, z, y) \text { holds iff } \rho(F(x, z, y), G(x, z)) .
$$

Then $\left(\mathrm{QVR}_{\alpha}\right)$ becomes the generalized quasi-variational inclusion problem:

Find $\bar{x} \in A$ such that $\bar{x} \in K_{1}(\bar{x})$ and $(y, z) \alpha K_{2}(\bar{x}) \times T(\bar{x})$ satisfying

$$
\rho(F(\bar{x}, z, y), G(\bar{x}, z)) \text {. }
$$

(III) If we let $A, D, B, X, Y, Z, K_{1}, K_{2}, T$ be as in $\left(\mathrm{QVR}_{\alpha}\right)$ and $F: A \times D \times B \rightarrow 2^{Z}$, $C: A \rightarrow 2^{Z}$ be multifunctions such that $C(x)$ is a closed convex cone with int $C(x) \neq \emptyset$, the relation $R$ is defined as follows:

$$
R(x, z, y) \text { holds iff } \rho(F(x, z, y), C(x)) \text {. }
$$

Then $\left(\mathrm{QVR}_{\alpha}\right)$ becomes the generalized vector quasi-equilibrium problem:

Find $\bar{x} \in A$ such that $\bar{x} \in K_{1}(\bar{x})$ and $(y, z) \alpha K_{2}(\bar{x}) \times T(\bar{x})$ satisfying

$$
\rho(F(\bar{x}, z, y), C(\bar{x})) .
$$

(IV) If we let $A, D, B, X, Y, Z, K_{1}, K_{2}$ be as in $\left(\mathrm{QVR}_{\alpha}\right), f: A \times D \times B \rightarrow Z$ be a vector function, and $C: A \rightarrow 2^{Z}$ be a multifunction such that $C(x)$ is a closed convex cone with int $C(x) \neq \emptyset$, the relation $R$ is defined as follows:

$$
R(x, z, y) \text { holds iff } f(x, z, y) \in C(x) \text {. }
$$

Then $\left(\mathrm{QVR}_{\alpha}\right)$ becomes the vector quasi-equilibrium problem:

Find $\bar{x} \in A$ such that $\bar{x} \in K_{1}(\bar{x})$ and $(y, z) \alpha K_{2}(\bar{x}) \times T(\bar{x})$ satisfying

$$
f(\bar{x}, z, y) \in C(\bar{x}) .
$$

(V) If we let $D, A=B, X=Y, Z, K_{1}, K_{2}, T$ be as in $\left(\mathrm{QVR}_{\alpha}\right), L(X, Z)$ be the space of all linear continuous operators from $X$ to $Z$ and $H: L(X, Z) \rightarrow L(X, Z), Q: A \times A \rightarrow X$, $F: A \times A \rightarrow Z$ be continuous single-valued mappings, $C: A \rightarrow 2^{Z}$ be a multifunction such that $C(x)$ is a closed convex cone with $\operatorname{int} C(x) \neq \emptyset$, the relation $R$ is defined as follows:

$$
R(x, z, y) \text { holds iff }\langle H(z), Q(y, x)\rangle+F(y, x) \in C(x) .
$$

Then $\left(\mathrm{QVR}_{\alpha}\right)$ becomes the generalized mixed vector quasi-variational inequality problem:

Find $\bar{x} \in A$ such that $\bar{x} \in K_{1}(\bar{x})$ and $(y, z) \alpha K_{2}(\bar{x}) \times T(\bar{x})$ satisfying

$$
\langle H(z), Q(y, \bar{x})\rangle+F(y, \bar{x}) \in C(\bar{x}) .
$$


Definition 1 ([22, 23]) Let $X, Y$ be two topological vector spaces, $A$ be a nonempty subset of $X$ and $F: A \rightarrow 2^{Z}$ be a multifunction.

(i) $F$ is said to be lower semicontinuous (lsc) at $x_{0} \in A$ if $F\left(x_{0}\right) \cap U \neq \emptyset$ for some open set $U \subseteq Y$ implies the existence of a neighborhood $N$ of $x_{0}$ such that $F(x) \cap U \neq \emptyset$, $\forall x \in N$. $F$ is said to be lower semicontinuous in $A$ if it is lower semicontinuous at all $x_{0} \in A$.

(ii) $F$ is said to be upper semicontinuous (usc) at $x_{0} \in A$ if for each open set $U \supseteq F\left(x_{0}\right)$, there is a neighborhood $N$ of $x_{0}$ such that $U \supseteq F(x), \forall x \in N$. $F$ is said to be upper semicontinuous in $A$ if it is upper semicontinuous at all $x_{0} \in A$.

(iii) $F$ is said to be continuous in $A$ if it is both lsc and usc in $A$.

(iv) $F$ is said to be closed if $\operatorname{Graph}(F)=\{(x, y): x \in A, y \in F(x)\}$ is a closed subset in $A \times Y$.

Definition 2 ([22]) Let $X, Y$ be two topological vector spaces, $A$ be a nonempty subset of $X, F: A \rightarrow 2^{Z}$ be a multifunction and $C \subset Y$ be a nonempty closed convex cone.

(i) $F$ is called upper $C$-continuous at $x_{0} \in A$ if for any neighborhood $U$ of the origin in $Y$, there is a neighborhood $V$ of $x_{0}$ such that

$$
F(x) \subset F\left(x_{0}\right)+U+C, \quad \forall x \in V .
$$

(ii) $F$ is called lower $C$-continuous at $x_{0} \in A$ if for any neighborhood $U$ of the origin in $Y$, there is a neighborhood $V$ of $x_{0}$ such that

$$
F\left(x_{0}\right) \subset F(x)+U-C, \quad \forall x \in V .
$$

Definition 3 ([23]) Let $X$ and $Y$ be two topological vector spaces and $A$ be a nonempty convex subset of $X$. A set-valued mapping $F: A \rightarrow 2^{Y}$ is said to be properly $C$-quasiconvex if for any $x, y \in A$ and $t \in[0,1]$, we have

$$
\begin{aligned}
& \text { either } F(x) \subset F(t x+(1-t) y)+C \\
& \text { or } F(y) \subset F(t x+(1-t) y)+C .
\end{aligned}
$$

Lemma 4 ([23]) Let $X, Y$ be two topological vector spaces, $A$ be a nonempty convex subset of $X$ and $F: A \rightarrow 2^{Y}$ be a multifunction.

(i) If $F$ is upper semicontinuous at $x_{0} \in A$ with closed values, then $F$ is closed at $x_{0} \in A$.

(ii) If $F$ is closed at $x_{0} \in A$ and $Y$ is compact, then $F$ is upper semicontinuous at $x_{0} \in A$.

(iii) If $F$ has compact values, then $F$ is usc at $x_{0} \in A$ if and only if, for each net $\left\{x_{\alpha}\right\} \subseteq A$ which converges to $x_{0} \in A$ and for each net $\left\{y_{\alpha}\right\}$ with $y_{\alpha} \subseteq F\left(x_{\alpha}\right)$, there are $y_{0} \in F\left(x_{0}\right)$ and a subnet $\left\{y_{\beta}\right\}$ of $\left\{y_{\alpha}\right\}$ such that $y_{\beta} \rightarrow y_{0}$.

Definition 5 ([24]) Let $X$ be a topological space. A subset $A$ of $X$ is called contractible at $x_{0} \in A$, if there is a continuous $F: A \times[0,1] \rightarrow A$ such that $F(z, 0)=z$ for all $z \in A$ and $F(z, 1)=x_{0}$ for all $z \in A$.

A topological space $X$ is said to be acyclic if all of its reduced Čech homology groups over the rationals vanish. In particular, each contractible space is acyclic, and thus any 
nonempty convex or star-shaped set is acyclic. Moreover, by the definition of a contractible set, we see that each convex space is contractible.

We now have the following fixed-point theorem in locally G-convex spaces given by Yuan [25] which is a generalization of the Fan-Glickberg-type fixed-point theorem for an upper semicontinuous set-valued mapping with nonempty closed acyclic values.

Theorem 6 ([25], Theorem 2.1) Let X be a compact locally G-convex space and $F: X \rightarrow$ $2^{X}$ be an upper semicontinuous set-valued mapping with nonempty closed acyclic values. Then $F$ has a fixed-point; that is, there exists an $x^{*} \in X$ such that $x^{*} \in F\left(x^{*}\right)$.

\section{Existence of solutions}

In this section, we apply the Kakutani-Fan-Glicksberg fixed-point theorem for upper semicontinuous set-valued mapping with nonempty closed acyclic values to establish sufficient conditions for the existence of a solution set of generalized quasi-variational relation problems. Moreover, the closedness of the solution set for these problems is obtained.

Definition 7 Let $X$ be a topological vector space, $A$ be a nonempty convex subset of $X$ and $R(x)$ be a relation linking $x \in A$. We say that $R$ is quasiconvex at $x_{0} \in A$ if $\forall x_{1}, x_{2} \in A$, $\forall \lambda \in[0,1]$ such that $R\left(x_{1}\right)$ holds and $R\left(x_{2}\right)$ holds, we have

$$
R\left(\lambda x_{1}+(1-\lambda) x_{2}\right) \text { holds. }
$$

$R$ is said to be quasiconvex in $A$ if it is quasiconvex at all $x_{0} \in A$.

Remark 8 In the Definition 7, if we let $X=A=\mathbb{R}$, and let mapping $F: \mathbb{R} \rightarrow \mathbb{R}$, then the relation $R$ defined by $R(x)$ holds iff $F(x) \subseteq \mathbb{R}_{-}$. We have $\forall x_{1}, x_{2} \in A, \forall \lambda \in[0,1]$, if $F\left(x_{1}\right) \leq 0$, $F\left(x_{2}\right) \leq 0$, then $F\left((1-\lambda) x_{1}+\lambda x_{2}\right) \leq 0$. This means that $R$ is modified 0-level quasiconvex, since the classical quasiconvexity says that $\forall x_{1}, x_{2} \in A, \forall \lambda \in[0,1]$,

$$
F\left((1-\lambda) x_{1}+\lambda x_{2}\right) \leq \max \left\{F\left(x_{1}, F\left(x_{2}\right)\right\} .\right.
$$

Theorem 9 Assume for the problem $\left(Q V R_{\alpha}\right)$ that

(i) $K_{1}$ is upper semicontinuous in $A$ with nonempty closed contractible values, and $K_{2}$ is lower semicontinuous $A$ with nonempty closed values;

(ii) $T$ is upper semicontinuous in $A$ with nonempty closed acyclic values if $\alpha=\mathrm{w}$ (or $\alpha=\mathrm{m})$ and lower semicontinuous in $A$ with nonempty acyclic values if $\alpha=\mathrm{s}$;

(iii) for all $(x, z) \in A \times D, R\left(x, z, K_{2}(x)\right)$ holds;

(iv) for all $(z, y) \in D \times B, R(\cdot, z, y)$ is quasiconvex in $A$;

(v) the set $\{(x, z, y) \in A \times D \times B: R(x, z, y)$ holds $\}$ is closed.

Then, the $\left(Q V R_{\alpha}\right)$ has a solution, i.e., there exist $\bar{x} \in A$ such that $\bar{x} \in K_{1}(\bar{x})$ and $(y, z) \alpha K_{2}(\bar{x}) \times$ $T(\bar{x})$ satisfying

$$
R(\bar{x}, z, y) \text { holds. }
$$


Proof Since $\alpha=\{\mathrm{w}, \mathrm{m}, \mathrm{s}\}$, we have in fact three cases. However, the proof techniques are similar. We present only the proof for the case where $\alpha=\mathrm{m}$.

Indeed, for all $(x, z) \in A \times D$, define a set-valued mapping: $\Psi_{m}: A \times D \rightarrow 2^{A}$ by

$$
\Psi_{m}(x, z)=\left\{a \in K_{1}(x): R(a, z, y) \text { holds, } \forall y \in K_{2}(x)\right\} .
$$

Since for any $x \in A, K_{1}(x), K_{2}(x)$ are nonempty. Thus, by assumption (iii), we have $\Psi_{m}(x, z) \neq \emptyset$.

(I) We show that $\Psi_{m}(x, z)$ is acyclic.

Since every contractible set is acyclic, it is enough to show that $\Psi_{m}(x, z)$ is contractible. Let $a \in \Psi_{m}(x, z)$, thus $a \in K_{1}(x)$ and $R(a, z, y)$ holds, $\forall y \in K_{2}(x)$. Since $K_{1}(x)$ is contractible, there exists a continuous mapping $f: K_{1}(x) \times[0,1] \rightarrow K_{1}(x)$ such that $f(b, 0)=b$ for all $b \in K_{1}(x)$ and $f(b, 1)=a$ for all $b \in K_{1}(x)$. Now, we set $F(b, \lambda)=\lambda a+(1-\lambda) h(b, \lambda)$ for all $(b, \lambda) \in \Psi_{m}(x, z) \times[0,1]$. Then $F$ is a continuous mapping, and we see that $F(b, 0)=b$ for all $b \in \Psi_{m}(x, z)$ and $F(b, 1)=a$ for all $b \in \Psi_{m}(x, z)$. Let $(b, \lambda) \in \Psi_{m}(x, z) \times[0,1]$, we need to prove that $F(b, \lambda) \in \Psi_{m}(x, z)$. Since $a, f(b, \lambda) \in K_{1}(x)$, and $K_{1}(x)$ is contractible, thus, for $a, f(b, \lambda) \in \Psi_{m}(x, z)$, it follows that

$$
R(a, z, y) \text { holds, } \quad \forall y \in K_{2}(x)
$$

and

$$
R(f(b, \lambda), z, y) \text { holds, } \quad \forall y \in K_{2}(x) .
$$

By (iv), $R(\cdot, z, y)$ is quasiconvex in $A$, we have

$$
R(\lambda a+(1-\lambda) f(b, \lambda), z, y) \text { holds, } \quad \forall y \in K_{2}(x)
$$

i.e., $F(b, \lambda) \in \Psi_{m}(x, z)$. Therefore, $\Psi_{m}(x, z)$ is contractible.

(II) We will prove $\Psi_{m}$ is upper semicontinuous in $A \times D$ with nonempty closed values.

Since $A$ is a compact set and $\Psi_{m}(x, z) \subset A$. Hence $\Psi(x, z)$ is compact. We need to show that $\Psi_{m}$ is a closed mapping. Indeed, let a net $\left\{\left(x_{n}, z_{n}\right)\right\} \subseteq A \times D$ such that $\left(x_{n}, z_{n}\right) \rightarrow(x, z) \in$ $A \times D$, and let $a_{n} \in \Psi_{m}\left(x_{n}, z_{n}\right)$ such that $a_{n} \rightarrow a_{0}$. Now, we need only prove that $a_{0} \in$ $\Psi_{m}(x, z)$. Since $a_{n} \in K_{1}\left(x_{n}\right)$ and $K_{1}$ is upper semicontinuous at $x \in A$ with nonempty closed values, by Lemma 4(i), we have $K_{1}$ is closed at $x \in A$, thus $a_{0} \in K_{1}(x)$. Suppose, to the contrary, $a_{0} \notin \Psi_{m}(x, z)$. Then $\exists y_{0} \in K_{2}(x)$ such that

$$
R\left(a_{0}, z, y_{0}\right) \text { does not hold. }
$$

By the lower semicontinuity of $K_{2}$, there is a net $\left\{y_{n}\right\}$ with $y_{n} \in K_{2}\left(x_{n}\right)$ such that $y_{n} \rightarrow y_{0}$. Since $a_{n} \in \Psi_{m}\left(x_{n}, z_{n}\right)$, we have

$$
R\left(a_{n}, z_{n}, y_{n}\right) \text { holds. }
$$

By the condition (v) and (2), we have 
There is a contradiction between (3) and (1). Thus, $a_{0} \in \Psi_{m}(x, z)$. Hence, $\Psi_{m}$ is upper semicontinuous in $A \times D$ with nonempty closed values.

(III) Now, we shall show that the solution set $\Sigma_{m}(R) \neq \emptyset$.

Define the set-valued mapping $H_{m}: A \times D \rightarrow 2^{A \times D}$ by

$$
H_{m}(x, z)=\left(\Psi_{m}(x, z), T(x)\right), \quad \forall(x, z) \in A \times D .
$$

Then $H_{m}$ is upper semicontinuous in $A \times D$ and $\forall(x, z) \in A \times D, H_{m}(x, z)$ is a nonempty closed convex subset of $A \times D$. By Theorem 6, there exists a point $(\bar{x}, \bar{z}) \in A \times D$ such that $(\bar{x}, \bar{z}) \in H_{m}(\bar{x}, \bar{z})$, that is,

$$
\bar{x} \in \Psi_{m}(\bar{x}, \bar{z}), \quad \bar{z} \in T(\bar{x}),
$$

which implies that there exists $\bar{x} \in A$ and $\bar{z} \in T(\bar{x})$ such that $\bar{x} \in K_{1}(\bar{x})$ and

$$
R(\bar{x}, \bar{z}, y) \text { holds, }
$$

i.e., $\bar{x} \in \Sigma_{m}(R)$.

(IV) Next, we prove that $\Sigma_{m}(R)$ is closed.

Let a net $\left\{x_{\alpha}, \alpha \in I\right\} \in \Sigma_{m}(R): x_{\alpha} \rightarrow x_{0}$. We need to prove that $x_{0} \in \Sigma_{m}(R)$. Indeed, by the lower semicontinuity of $K_{2}$, for any $y_{0} \in K_{2}\left(x_{0}\right)$, there exists $y_{n} \in K_{2}\left(x_{\alpha}\right)$ such that $y_{n} \rightarrow y_{0}$. As $x_{\alpha} \in \Sigma_{m}(R)$, there exists $z_{\alpha} \in T\left(x_{\alpha}\right)$ such that

$$
R\left(x_{\alpha}, z_{\alpha}, y_{\alpha}\right) \text { holds. }
$$

Since $K_{1}$ is upper semicontinuous with nonempty closed values, by Lemma 4(i), we have $K_{1}$ is closed. Thus, $x_{0} \in K_{1}\left(x_{0}\right)$. Since $T$ is upper semicontinuous in $A$ and $T\left(x_{0}\right)$ is compact, there exists $z_{0} \in T\left(x_{0}\right)$ such that $z_{\alpha} \rightarrow z_{0}$. By the condition (v), we have

$$
R\left(x_{0}, z_{0}, y_{0}\right) \text { holds. }
$$

This means that $x_{0} \in \Sigma_{m}(R)$. Thus $\Sigma_{m}(R)$ is a closed set.

Remark 10 If we let $A=B, D, X=Y, Z, K_{1}=K_{2}=K, T, R, \alpha=\mathrm{m}$ as in $\left(\mathrm{QVR}_{\alpha}\right), F: A \times$ $D \times A \rightarrow 2^{Z}$ be a multifunction and $C \subset Z$ be a nonempty closed convex cone, the relation $R$ is defined as follows:

$$
R(x, z, y) \text { holds iff } F(x, z, y) \subset C,
$$

and

$$
R(x, z, y) \text { holds iff } F(x, z, y) \not \subset C .
$$

Then, $\left(\mathrm{QVR} \mathrm{R}_{\alpha}\right)$ becomes the generalized strong vector quasi-equilibrium problem of type (I) and (II) (in short, (GSVQEP I) and (GSVQEP II)) studied in [17]. 
(GSVQEP I): Find $\bar{x} \in A$ and $\bar{z} \in T(\bar{x})$ such that $\bar{x} \in K(\bar{x})$ and

$$
F(\bar{x}, \bar{z}, y) \subset C, \quad \text { for all } y \in K(\bar{x})
$$

and

(GSVQEP II): Find $\bar{x} \in A$ and $\bar{z} \in T(\bar{x})$ such that $\bar{x} \in K(\bar{x})$ and

$$
F(\bar{x}, \bar{z}, y) \not \subset C, \quad \text { for all } y \in K(\bar{x}) \text {. }
$$

The following example shows that all the assumptions of Theorem 9 are satisfied, but Theorem 3.1 in [17] does not work. The reason is that $F$ is not lower $(-C)$-continuous.

Example 11 Let $X=Y=Z=\mathbb{R}, A=B=D=[0,1], C=\mathbb{R}_{+}, K_{1}(x)=K_{2}(x)=[0,1]$ and

$$
T_{1}(x)=T_{2}(x)= \begin{cases}{[0,1]} & \text { if } x_{0}=\frac{1}{2} \\ {\left[0, \frac{1}{2}\right]} & \text { otherwise }\end{cases}
$$

and

$$
F(x, z, y)= \begin{cases}{\left[\frac{1}{2}, 1\right]} & \text { if } x_{0}=z_{0}=y_{0}=\frac{1}{2} \\ {[1,2]} & \text { otherwise }\end{cases}
$$

We let the relation $R$ be defined by $R(x, z, y)$ holds iff $F(x, z, y) \subseteq \mathbb{R}_{+}$. We can show that all the assumptions of Theorem 9 are satisfied. However, $F$ is not lower $(-C)$-continuous at $x_{0}=\frac{1}{2}$. Also, Theorem 3.1 in [17] does not work.

The following example shows that all the assumptions of Theorem 9 are satisfied, but Theorem 3.1 in [17] is not fulfilled. The reason is that $F$ is not upper $C$-continuous.

Example 12 Let $A, B, D, X, Y, Z, K, C$ be as in Example 11 and $T(x)=\{z\}$ and

$$
F(x, z, y)= \begin{cases}{\left[1, \frac{5}{2}\right]} & \text { if } x_{0}=z_{0}=y_{0}=\frac{1}{2} \\ {\left[\frac{1}{5}, \frac{3}{4}\right]} & \text { otherwise. }\end{cases}
$$

We let the relation $R$ be defined by $R(x, z, y)$ holds iff $F(x, z, y) \subseteq \mathbb{R}_{+}$. It is easy to check that all the assumptions of Theorem 9 are satisfied. So, $\left(\mathrm{QVR}_{\alpha}\right)$ has a solution. However, $F$ is not upper $C$-continuous at $x_{0}=\frac{1}{2}$. Also, Theorem 3.1 in [17] does not work.

The following example shows that all assumptions of Theorem 9 are satisfied, but Theorem 3.1 in [17] is not fulfilled. The reason is that $F$ is not $C$-quasiconvex.

Example 13 Let $A, B, D, X, Y, Z, K, C, T$ be as in Example 12 and

$$
F(x, z, y)= \begin{cases}{[1,2]} & \text { if } x_{0}=z_{0}=y_{0}=\frac{1}{2} \\ {\left[\frac{1}{2}, 1\right]} & \text { otherwise }\end{cases}
$$


We let the relation $R$ be defined by $R(x, z, y)$ holds iff $F(x, z, y) \subseteq \mathbb{R}_{+}$. It is easy to check that all the assumptions of Theorem 9 are satisfied. However, $F$ is not $C$-quasiconvex at $x_{0}=\frac{1}{2}$. Thus, it gives also cases where Theorem 9 can be applied but Theorem 3.1 in [17] does not work.

If we let $X, Y, Z$ be real locally convex Hausdorff topological vector spaces, then we have the following corollary.

Corollary 14 Assume for problem $\left(Q V R_{\alpha}\right)$ that

(i) $K_{1}$ is upper semicontinuous in $A$ with nonempty closed convex values, and $K_{2}$ is lower semicontinuous A with nonempty closed values;

(ii) $T$ is upper semicontinuous in $A$ with nonempty closed convex values if $\alpha=\mathrm{w}$ (or $\alpha=\mathrm{m}$ ) and lower semicontinuous in $A$ with nonempty convex values if $\alpha=\mathrm{s}$;

(iii) for all $(x, z) \in A \times D, R\left(x, z, K_{2}(x)\right)$ holds;

(iv) for all $(z, y) \in D \times B, R(\cdot, z, y)$ is quasiconvex in $A$;

(v) the set $\{(x, z, y) \in A \times D \times B: R(x, z, y)$ holds $\}$ is closed.

Then the $\left(Q V R_{\alpha}\right)$ has a solution, i.e., there exist $\bar{x} \in A$ such that $\bar{x} \in K_{1}(\bar{x})$ and $(y, z) \alpha K_{2}(\bar{x}) \times$ $T(\bar{x})$ satisfying

$R(\bar{x}, z, y)$ holds.

Moreover, the solution set of the $\left(Q V R_{\alpha}\right)$ is closed.

\section{Remark 15}

(i) If we let $X, Y, Z$ be real locally convex Hausdorff topological vector spaces, then (GSVQEP I) becomes the problem (GSVQEP) studied in [15].

(ii) If $A=B, X=Y, Z, K_{1}=K_{2}=K, R$ as in $\left(\mathrm{QVR}_{\alpha}\right)$ and $T(x)=\{z\}, F: A \times A \rightarrow 2^{Z}$ is a multifunction, $C \subset Z$ is a nonempty closed convex cone, the relation $R$ is defined as follows:

$$
R(x, z, y) \text { holds } \quad \text { iff } \quad F(x, y) \subset C \text {. }
$$

Then $\left(\mathrm{QVR}_{\alpha}\right)$ becomes strong vector quasi-equilibrium problem (in short, (SVQEP)) studied in [18].

Find $\bar{x} \in A$ such that $\bar{x} \in K(\bar{x})$ and

$$
F(\bar{x}, y) \subset C, \quad \text { for all } y \in K(\bar{x}) .
$$

\section{Remark 16}

(i) Corollary 14 improves and extends Theorem 3.1 in [15] and Theorem 3.3 in [18].

(ii) Theorem 9 improves and extends Theorems 3.1 and 3.3 in [17].

\section{Stability}

In this section, we discuss the stability of the solutions for $\left(\mathrm{QVR}_{\alpha}\right)$. Throughout this section, let $X, Y, Z$ be Banach spaces, $N$ be a real locally $G$-convex Hausdorff topological vector space. Let $A \subset X, B \subset Y$ and $D \subset Z$ be nonempty compact convex subsets, 
$K_{1} \equiv K_{2} \equiv K: A \rightarrow 2^{A}, T: A \rightarrow 2^{B}$ be multifunctions, and $R(x, z, y)$ be a relation linking $x \in A, z \in D$ and $y \in B$. Now, we let

$$
\Xi:=\{(T, K) \mid T \text { is upper semicontinuous in } A \text { with nonempty closed acyclic values, }
$$

if $\alpha=\mathrm{w}$ (or $\alpha=\mathrm{m}$ ), and lower semicontinuous in $A$ with nonempty acyclic

values, if $\alpha=\mathrm{s}$, and $\mathrm{K}$ is continuous with nonempty closed contractible values\}.

Let $E_{1}, E_{2}$ be compact sets in a normed space. Recall that the Hausdorff metric is defined by

$$
H\left(E_{1}, E_{2}\right):=\max \left\{H^{\prime \prime}\left(E_{1}, E_{2}\right), H^{\prime \prime}\left(E_{2}, E_{1}\right)\right\}
$$

where $H^{*}\left(E_{1}, E_{2}\right):=\sup _{e_{1} \in E_{1}} d\left(e_{1}, E_{2}\right)$ and $d\left(e_{1}, E_{2}\right):=\inf _{e_{2} \in E_{2}}\left\|e_{1}-e_{2}\right\|$.

For $(T, K),\left(T^{\prime}, K^{\prime}\right) \in \Xi$, define

$$
\xi\left((T, K),\left(T^{\prime}, K^{\prime}\right)\right):=\sup _{x \in A} H_{1}\left(T(x), T^{\prime}(x)\right)+\sup _{x \in A} H_{2}\left(K(x), K^{\prime}(x)\right),
$$

where $H_{1}, H_{2}$ are the appropriate Hausdorff metrics. Obviously, $(\Xi, \xi)$ is a metric space.

Assume that $R$ satisfies the conditions of Theorem 9. Then for each $(T, K) \in \Xi,\left(\mathrm{QVR}_{\alpha}\right)$ has a solution $\bar{x}, i . e$., there exists $\bar{x} \in A$ such that $\bar{x} \in K(\bar{x})$ and $(y, \bar{z}) \alpha K(\bar{x}) \times T(\bar{x})$ satisfying

$R(\bar{x}, \bar{z}, y)$ holds.

For $(T, K) \in \Xi$, let

$$
\begin{aligned}
\Theta_{\alpha}(T, K):= & \{\bar{x} \in A \text { such that } \bar{x} \in K(\bar{x}) \\
& \text { and }(y, \bar{z}) \alpha K(\bar{x}) \times T(\bar{x}) \text { satisfying } R(\bar{x}, \bar{z}, y) \text { holds }\} .
\end{aligned}
$$

Then $\Theta_{\alpha}(T, K) \neq \emptyset$, and so $\Theta_{\alpha}(T, K)$ defines a set-valued mapping from $\Xi$ into $A$.

Lemma 17 ([26]) Let $Z$ be a metric space and let $M, M_{n}(n=1,2, \ldots)$ be compact sets in $Z$. Suppose that for any open set $O \supset M$, there exists $n_{0}$ such that $M_{n} \subset O, \forall n \geq n_{0}$. Then any sequence $\left\{x_{n}\right\}$ satisfying $x_{n} \in M_{n}$ has a convergent subsequence with limit in $M$.

Theorem $18 \Theta_{\alpha}: \Xi \rightarrow 2^{A}$ is upper semicontinuous with compact values.

Proof Similar arguments can be applied to three cases. We present only the proof for the cases where $\alpha=\mathrm{m}$. Indeed, since $A$ is compact, we need only show that $\Theta_{m}$ is a closed mapping. Let a sequence $\left\{\left(T_{n}, K_{n}, x_{n}\right)\right\} \subset \operatorname{Graph}\left(\Theta_{m}\right)$ be given such that $\left(T_{n}, K_{n}, x_{n}\right) \rightarrow$ $\left(T, K, x_{0}\right)$. We now show that $\left\{\left(T, K, x_{0}\right)\right\} \subset \operatorname{Graph}\left(\Theta_{m}\right)$.

For any $n$, since $x_{n} \in \Theta_{m}\left(T_{n}, K_{n}\right)$, we have that $x_{n} \in K_{n}\left(x_{n}\right)$ and $\exists z_{n} \in T_{n}\left(x_{n}\right), \forall y_{n} \in K_{n}\left(x_{n}\right)$ such that

$$
R\left(x_{n}, z_{n}, y_{n}\right) \text { holds. }
$$


For any open set $O \supset T\left(x_{0}\right)$, since $T\left(x_{0}\right)$ is a compact set, there exists $\varepsilon>0$ such that

$$
\left\{z \in Y: d\left(z, T\left(x_{0}\right)\right)<\varepsilon\right\} \subset O
$$

where $d\left(z, T\left(x_{0}\right)\right)=\inf _{z^{\prime} \in T\left(x_{0}\right)}\left\|z-z^{\prime}\right\|$.

Since $\xi\left(\left(T_{n}, K_{n}\right),(T, K)\right) \rightarrow 0, x_{n} \rightarrow x_{0}$ and $T$ is upper semicontinuous at $x_{0}, \exists n_{0}$ such that

$$
\begin{aligned}
& \sup _{x \in A} H_{1}\left(T_{n}(x), T(x)\right)<\frac{\varepsilon}{2}, \\
& T\left(x_{n}\right) \subset\left\{z \in Y: d\left(z, T\left(x_{0}\right)\right)<\frac{\varepsilon}{2}\right\}, \quad \forall n \geq n_{0} .
\end{aligned}
$$

From (5), (6) and (7), we have

$$
T\left(x_{n}\right) \subset\left\{z \in Y: d\left(z, T\left(x_{0}\right)\right)<\frac{\varepsilon}{2}\right\} \subset\left\{z \in Y: d\left(z, T\left(x_{0}\right)\right)<\varepsilon\right\} \subset O, \quad \forall n \geq n_{0}
$$

Since $T\left(x_{0}\right) \subset O$ and $z_{n} \in T_{n}\left(x_{n}\right)$, we can apply Lemma 17 . There exists a subsequence $\left\{z_{n_{k}}\right\}$ of $\left\{z_{n}\right\}$ such that $\left\{z_{n_{k}}\right\}$ convergent to $z_{0}$, it follows that $z_{0} \in T\left(x_{0}\right)$. By using the same argument as above, we can show that $x_{0} \in K\left(x_{0}\right)$.

Next, we need only show that $R\left(x_{0}, z_{0}, y_{0}\right)$ holds. Since $x_{n} \rightarrow x_{0}$ and $K$ is upper semicontinuous at $x_{0}, K\left(x_{0}\right)$ is closed, there exists $y_{0} \in K\left(x_{0}\right)$ such that $y_{n} \rightarrow y_{0}$ (taking a subsequence if necessary).

Since $\xi\left(\left(T_{n}, K_{n}\right),(T, K)\right) \rightarrow 0$, we can chose a subsequence $\left\{K_{n_{k}}\right\}$ of $\left\{K_{n}\right\}$ such that

$$
\sup _{x \in A} H_{2}\left(K_{n_{k}}(x), K(x)\right)<\frac{1}{k}
$$

Thus,

$$
H_{2}\left(K_{n_{k}}\left(x_{n_{k}}\right), K\left(x_{n_{k}}\right)\right)<\frac{1}{k}
$$

This implies that there exist $t_{n_{k}}^{\prime} \in K_{n_{k}}\left(x_{n_{k}}\right), k=1,2, \ldots$ such that

$$
\left\|t_{n_{k}}^{\prime}-t_{n_{k}}\right\|<\frac{1}{k}
$$

As

$$
\left\|t_{n_{k}}^{\prime}-y_{0}\right\| \leq\left\|t_{n_{k}}^{\prime}-t_{n_{k}}\right\|+\left\|t_{n_{k}}-y_{0}\right\|<\frac{1}{k}+\left\|t_{n_{k}}-y_{0}\right\| \rightarrow 0
$$

and so we have $t_{n_{k}}^{\prime} \rightarrow y_{0}$. Since $x_{n_{k}} \in K_{n_{k}}\left(x_{n_{k}}\right), z_{n_{k}} \in T_{n_{k}}\left(x_{n_{k}}\right)$ and $t_{n_{k}}^{\prime} \in K_{n_{k}}\left(x_{n_{k}}\right)$, applying (5), we have

$$
R\left(x_{n_{k}}, z_{n_{k}}, t_{n_{k}}^{\prime}\right) \text { holds. }
$$

Assumption (v) yields that

$$
R\left(x_{0}, z_{0}, y_{0}\right) \text { holds. }
$$


Since $x_{0} \in K\left(x_{0}\right)$ and $z_{0} \in T\left(x_{0}\right)$ and (10) yields that $\left(T, K, x_{0}\right) \in \operatorname{Graph}\left(\Theta_{m}\right)$ and so $\operatorname{Graph}\left(\Theta_{m}\right)$ is closed. Therefore, $\Theta_{m}$ is closed. Since $A$ is a compact set and $\Theta_{m}(T, K) \subset A$. Hence $\Theta_{m}$ has a compact valued mapping.

Remark 19 Theorem 18 improves and extends Theorems 3.1 and 3.3 in [17], Theorem 3.1 in $[15]$.

\section{Competing interests}

The author declares that he has no competing interests.

\section{Acknowledgements}

The author thanks the two anonymous referees for their valuable remarks and suggestions, which helped him to improve the article considerably.

Received: 22 July 2012 Accepted: 4 September 2012 Published: 19 September 2012

\section{References}

1. Balaj, M, Lin, LJ: Generalized variational relation problems with applications. J. Optim. Theory Appl. 148, 1-13 (2011)

2. Hung, NV: Continuity of solutions for parametric generalized quasivariational relation problems. Fixed Point Theory Appl. 2012, 102 (2012)

3. Khanh, PQ, Luc, DT: Stability of solutions in parametric variational relation problems. Set-Valued Anal. 16, 1015-1035 (2008)

4. Luc, DT, Sarabi, E, Soubeyran, A: Existence of solutions in variational relation problems without convexity. J. Math. Anal. Appl. 364, 544-555 (2010)

5. Fu, JY: Symmetric vector quasiequilibrium problems. J. Math. Anal. Appl. 285, 708-713 (2003)

6. Kien, BT, Huy, NQ, Wong, NC: On the solution existence of generalized vector quasi-equilibrium problems with discontinuous multifunctions. Taiwan. J. Math. 13, 757-775 (2009)

7. Lin, Z, Yang, $\mathrm{H}, \mathrm{Yu}$, J: On existence and essential components of the solution set for the system of vector quasi-equilibrium problems. Nonlinear Anal. 63, 2445-2452 (2005)

8. Lin, Z, Yu, J: The existence of solutions for the system of generalized vector quasi-equilibrium problems. Appl. Math. Lett. 18, 415-422 (2005)

9. Lin, L, Liu, YH: Existence theorems for systems of generalized vector quasiequilibrium problems and optimization problems. J. Optim. Theory Appl. 130, 461-475 (2006)

10. Hai, NX, Khanh, PQ: Existence of solution to general quasiequilibrium problem and applications. J. Optim. Theory Appl. 133, 317-327 (2007)

11. Hai, NX, Khanh, PQ: Systems of set-valued quasivariational inclusion problems. J. Optim. Theory Appl. 135, 55-67 (2007)

12. Liu, QY, Long, XJ, Huang, NJ: Connectedness of the sets of weak efficient solutions for generalized vector equilibrium problems. Math. Slovaca 62, 123-136 (2012)

13. Long, XJ, Peng, JW: Connectedness and compactness of weak efficient solutions for vector equilibrium problems. Bull. Korean Math. Soc. 48, 1225-1233 (2011)

14. Long, XJ, Huang, YQ, Peng, ZY: Optimality conditions for the Henig efficient solution of vector equilibrium problems with constraints. Optim. Lett. 5, 717-728 (2011)

15. Long, XJ, Huang, NJ, Teo, KL: Existence and stability of solutions for generalized strong vector quasi-equilibrium problems. Math. Comput. Model. 47, 445-451 (2008)

16. Song, QQ, Wang, LS: The existence of solutions for the system of vector quasi-equilibrium problems in topological order spaces. Comput. Math. Appl. 62, 1979-1983 (2011)

17. Plubtieng, S, Sitthithakerngkiet, K: Existence result of generalized vector quasiequilibrium problems in locally G-convex spaces. Fixed Point Theory Appl. 2011, Article ID 967515 (2011)

18. Yang, Y, Pu, YJ: On the existence and essential components for solution set for system of strong vector quasiequilibrium problems. J. Glob. Optim. (2011). doi:10.1007/s10898-011-9830-y

19. Hou, SH, Gong, XH, Yang, XM: Existence and stability of solutions for generalized Ky Fan inequality problems with trifunctions. J. Optim. Theory Appl. 146, 387-398 (2010)

20. Lin, Z: Existence of solutions to the system of generalized implicit vector quasivariational inequality problems. Fixed Point Theory Appl. 2009, Article ID 654370 (2009)

21. Park, S, Kim, H: Admissible classes of multifunctions on generalized convex spaces. Proc. Coll. Nat. Sci. 18, 1-21 (1993)

22. Berge, C: Topological Spaces. Oliver \& Boyd, London (1963)

23. Luc, DT: Theory of Vector Optimization. Lecture Notes in Economics and Mathematical Systems. Springer, Berlin (1989)

24. Bardaro, C, Ceppitelli, R: Some further generalizations of Knaster-Kuratowski-Mazurkiewicz theorem and minimax inequalities. J. Math. Anal. Appl. 132, 484-490 (1988)

25. Yuan, GXZ: Fixed points of upper semicontinuous mappings in locally G-convex spaces. Bull. Aust. Math. Soc. 58, 469-478 (1998)

26. Yu, J: Essential weak efficient solution in multiobjective optimization problems. J. Math. Anal. Appl. 166, 230-235 (1992) 
doi:10.1186/1687-1812-2012-158

Cite this article as: Hung: Sensitivity analysis for generalized quasi-variational relation problems in locally G-convex spaces. Fixed Point Theory and Applications 2012 2012:158.

Submit your manuscript to a SpringerOpen ${ }^{\circ}$ journal and benefit from:

- Convenient online submission

- Rigorous peer review

- Immediate publication on acceptance

- Open access: articles freely available online

- High visibility within the field

- Retaining the copyright to your article

Submit your next manuscript at $\gg$ springeropen.com 